\title{
Encoding and Retrieval in Human Medial Temporal Lobes: An Empirical Investigation Using Functional Magnetic Resonance Imaging (fMRI)
}

\author{
R. J. Dolan, ${ }^{1,2 *}$ and P. F. Fletcher ${ }^{1}$ \\ ${ }^{1}$ The Wellcome Department of Cognitive Neurology, \\ Institute of Neurology, London, United Kingdom \\ ${ }^{2}$ The Royal Free Hospital Medical School, London, \\ United Kingdom
}

\begin{abstract}
The precise functional role of the hippocampus in human episodic memory is an unresolved question though it has recently been suggested that distinct medial temporal lobe (MTL) regions are involved in encoding and retrieval operations respectively. For example, a recent meta-analysis of positron emission tomography (PET) literature has suggested a rostral-caudal functional division in the medial temporal lobes (MTL), with rostral MTL mediating encoding and caudal MTL retrieval operations. However, a review of the combined PET and fMRI literature, reported in the present issue, while noting systematic discrepancies between PET and $\mathrm{FMRI}$, reaches a conclusion that posterior MTL is involved in encoding. Here we present fMRI data, from a modified artificial grammar learning paradigm, that examines two questions concerning the functional role of the hippocampus, and related MTL structures in episodic memory. Firstly, we test a hypothesis that anterior hippocampus is activated during encoding and that this response is greater for novel items. Secondly, we test whether increasing familiarity with stimulus material is associated with a posterior MTL neural response. 0 ur empirical findings support both hypotheses in that we demonstrate a left anterior hippocampal response sensitive to encoding demands and a posterior parahippocampal response sensitive to retrieval demands. Furthermore, we show that both anterior and posterior hippocampal responses are modulated to the degree to which stimuli can be assimilated into ameaningful rule-based framework. Hippocampus 1999;9:25-34. ㅇ 1999 Wiley-Liss, Inc.
\end{abstract}

KEY WORDS: hippocampus; parahippocampus; neuroimaging; artificial grammar

\section{INTRODUCTION}

The effects of localized brain damage provides compelling evidence that psychologically distinct forms of memory are dependent on the integrity of discrete anatomical brain systems (Scoville and Milner, 1957). In this respect, the clinical syndrome of medial temporal amnesia has been central in framing a concept of memory that suggests multiple dissociable domains (C ohen, 1984). Theobservations that medial temporal lobe(M TL) damage leads to a pervasive inability to remember new facts and events is now well established (Rempel-Clower et al., 1996). What remains a source of

Grant sponsor: The Wellcome Trust.

*Correspondence to: Raymond J Dolan, The Wellcome Department of Cognitive Neurology, Institute of N eurology, London WC1N 3BG. E-mail: r.dolan@fil.ion.ucl.ac.uk

Accepted 300 ctober 1998 controversy is whether separate anatomical regions within $M T L$ are specialized for distinct aspects and operations within what is termed declarative memory. Such functional specialization is suggested by observations that patients with damage limited to the hippocampal formation have more restricted deficits than patients with more extensive temporal lobe damage (Reed and Squire, 1998). Furthermore, damage to anterior hippocampus while leading to dramatic impairments in episodic memory is not incompatible with preservation of some aspects of declarative memory. For example, it is now reported that young children with hippocampal damage retain an ability to acquire facts and knowledge about the world (Vargha-Khadem et al., 1997). Thus, while the importance of M TL in episodic memory is beyond dispute, thequestion raised by these recent neuropsychological studies is whether distinct regions of hippocampus, and related MTL structures, are specialized for particular aspects of memory function.

While neuropsychological data have been crucial in identifying dissociable forms of memory, it is recognized that patient studies are problematic in isolating the precise components of memory affected by a lesion. For example, it is difficult to separate the effects of lesions on encoding or retrieval operations. In this respect functional neuroimaging provides an important advantage in so far as data can be acquired in an intact, normally functioning brain. An extensive neuroimaging literature, involving verbal and non-verbal material, has provided persuasive evidence for relative specialization in left and right prefrontal cortex for encoding and retrieval, respectively (Tulving et al., 1994a,b; Kapur et al., 1994; Shallice et al., 1994; Nyberg et al., 1996a,b). This putative asymmetry was not previously known from lesion-based data. Recent application of functional neuroimaging al so demonstrates that aside from localization it is feasible to perform experiments that allow precise specification of the psychological operations that are implemented in encoding and retrieval related regional activations (Rugg et al., 1996; Fletcher et al., 1998a,b). What is unclear from these data is whether hemispheric 
specialization at encoding and retrieval reflect encoding or retrieval processes per se or, as is more likely in our view, psychological operations that are preferentially engaged at these two stages of memory function.

In a previously reported finding, we sought to reconcile prefrontal and hippocampal involvement during auditory-verbal episodic memory encoding with a hypothesis that hippocampal activation reflects a response to relative novelty. In this study, that stimulus material consisted of word paired-associates comprising a category (e.g., Dog) and an exemplar (e.g., Boxer) presented auditorily. Left dorsolateral prefrontal cortex was sensitive to a manipulation of the association between category and exemplar. By contrast MTL, including hippocampus and parahippocamal regions, showed a striking responsiveness to novelty in the presented material (D olan and Fletcher, 1997). Thus, maximal hippocampal activation was seen in the condition where both category and exemplar were novel. These previously reported findings, and others (Tulving et al., 1994c, 1996; Stern et al., 1996; Gabrieli et al., 1997), suggest that a component of hippocampal processing at encoding relates to novelty detection.

In this paper, we use functional neuroimaging to address two questions regarding the role of $M T L$ in episodic memory. The first concerns the role of MTL structures, and in particular the hippocampus, in episodic memory encoding and retrieval. The second question concerns the type of processing implemented in the hippocampus during encoding and, specifically, whether this relates to novelty detection. To address these questions, we used a modified artificial grammar learning paradigm that involved subjects learning the grammatical status of letter strings according to predetermined rules. In the variant of the task used in our experiment, feedback related to task performance, was provided on a trial by trial basis. Consequently, this type of paradigm is particularly appropriate to study encoding and retrieval processes in so far as subjects have no prior knowledge of the grammar system but, over the course of an experiment, gradually acquire the underlying rules. Thus, in the early phases of learning, subjects' responses are to items that have no inherent meaning and, in this respect, are more novel than items presented later in the experiment. U sing a block design in which the same items are presented repeatedly, it is possible to modulate the degree to which the task requirements depends on episodic encoding and retrieval. Furthermore, over the course of the experiment subjects acquire knowledge of the rule system leading to a decrease in the degree to which reliance on episodic encoding and retrieval is necessary for task performance. Consequently, using this paradigm (see below for details) it is possible to assess the influences of encoding and retrieval against the background of an emerging knowledge structure that, over the course of the experiment, increasingly influences subjects' responses to the grammatical status of new items. In other words, this type of paradigm can be used to determine how psychological context (acquisition of a rule system) influences encoding and retrieval related neural responses.

\section{METHODS}

\section{Memory Task}

We used a finite state grammar system (see Fig. 1) to generate consonant strings under the constraint that all strings must consist of 4 letters. A total of 30 strings, mixed with 30 arbitrarily chosen non-grammatical lures, were presented over the course of the entire experiment. The experiment comprised 6 blocks (see Fig. 2) and within each block stimuli were presented on a screen, at a rate of one per 3.2 seconds, with a total of 10 consonant strings ( $50 \%$ grammatical; $50 \%$ lures). Subjects were required to respond to items by indicating with a key pad whether the item was grammatical or ungrammatical. Subjects received immediate visual feedback indicating whether their response was correct or incorrect. In a single block, the same 10 items were presented a total of 6 times. Consequently, responses to items within a block are strongly influenced by recollection of the outcome of previous responses to that item. In this respect, within-block effects are strongly dependent upon episodic memory as can also be seen in the behavioral data (see below). Each presentation epoch was followed by a sensorimotor control condition that consisted of serial visual presentation of either rows of the letter $\mathrm{P}$ or $\mathrm{N}$ signaling a right or left key-pad response, respectively. At the end of each block, a new block began and 10 new items (5 grammatical, 5 ungrammatical) were presented. Thus, in the early stage of the experiment, subjects had no knowledge of the underlying grammatical rules and responded on the basis of guesses and explicit knowledge of outcomes of previous responses to individual items. Across blocks, these responses are gradually modulated by the emergence of knowledge of grammatical rules. Six identically structured blocks were presented in total, with each successive block containing 10 new items (see Fig. 2 for details).

Behaviourial data were acquired on-line enabling characterization of rate of learning both within (corresponding to learning the grammatical status of individual items) and across blocks (corresponding to learning the underlying grammatical rules of the grammar system). The former clearly depends upon episodic memory in that to determine the within-block grammatical state of individual items, subjects rely on recollection of the outcome (correct or incorrect performance feedback provided on a trial by trial basis) of previous encounters with the item. By contrast, across-block effects reflect learning of underlying rules of the grammar system. Consequently, this across-block effect can be thought of as creating a dynamic knowledge context for learning. $N$ ote again that responses to individual exemplars early in the experiment are independent of rule knowledge and rely almost exclusively upon episodic memory. H owever, across blocks this context changes as subjects' judgment of the grammatical status of items is progressively influenced by an abstraction of underlying grammatical rules. Therefore, the design of our experiment means that across blocks there is modulation of episodic memory processes by the creation of an underlying knowledge base. In 


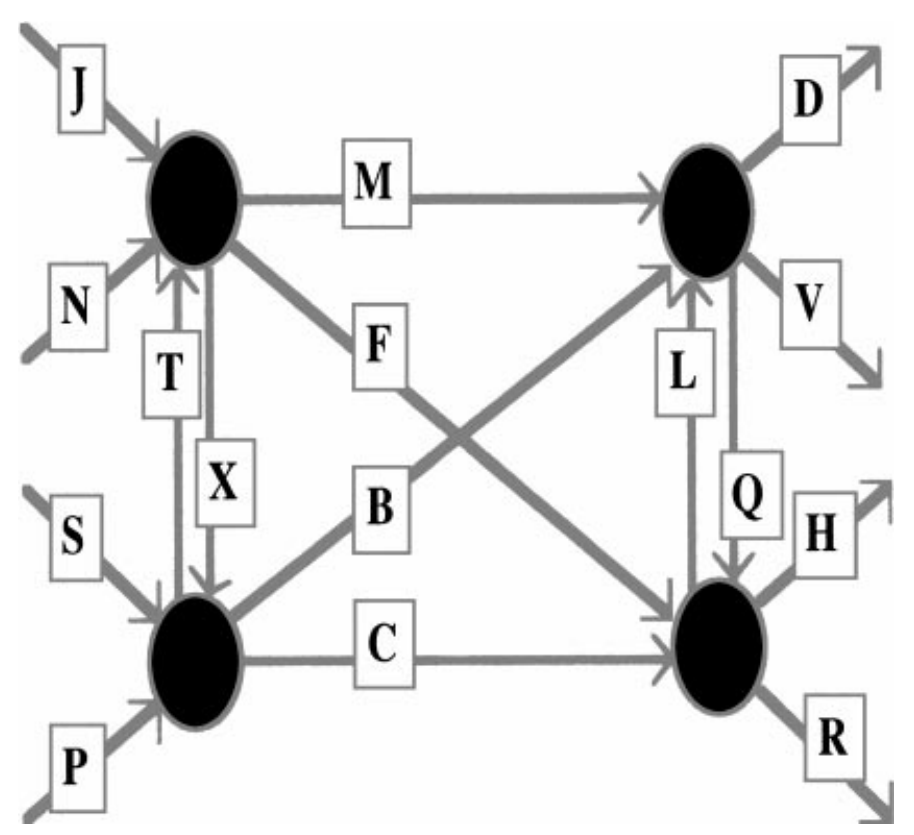

FIGURE 1. The finite-state grammar rule system (hidden from subjects throughout) used in the experiment. Grammatical items are produced by starting at the left and moving from node to successive node always following the direction of arrows. Every arrow traversed generates the attached letter. We added the constraint that all items consisted of 4 letters. Thus JMQH, STM V, and JFLV would all be deemed grammatical, whereas JQLD, SCHR, and JMFR would be deemed ungrammatical.

other words, correct responses to items presented in later blocks can be made by an abstraction of the underlying rules. Our experiment provides clear predictions in terms of hippocampal responseto learning, which can be framed as follows. A hippocampal response to novel individual items will attenuate with repeated exposure to these items (a within-block effect) and this response will itself be modulated as a function of abstraction of underlying grammatical rules (across-block effect). M ore simply, we predict a time by condition interaction in the hippocampus with respect to its response to novel exemplars of the grammar system.

\section{Functional Neuroimaging}

Informed consent was obtained from 4 subjects ( 2 male, 2 female; all right-handed; age range 26- 36 yrs; mean age 29 years). A Siemens VISI O N system (Siemens; Erlangen, Germany) operating at 2 Tesla was used to acquire both T1 anatomical and gradient-echo echo-planar T2* weighted image volumes with blood oxygenation level dependent (BOLD) contrast. For all subjects, data were acquired in 2 scanning sessions separated by a 5 -minute rest period. Aside from 6 "dummy" volumes, which were subsequently discarded to allow for $\mathrm{T} 1$ equilibration effects, a total of 360 functional volumes were acquired per subject. Volumes were acquired continuously every 6,400 ms. Each volume comprised sixty-four $3 \mathrm{~mm}$ axial slices with in-plane resolution $3 \times 3 \mathrm{~mm}$ positioned to cover the whole brain. The imaging time series was realigned, spatially normalized, and smoothed with a Gaussian kernel of $8 \mathrm{~mm}$ full width half maximum (Talairach and Tournoux, 1988; Friston et al., 1995a,b). Low frequency cosine waves modeled and removed subject-specific low frequency drifts in signal and the data were normalized for global effects by proportional scaling ( $\mathrm{H}$ olmes et al., 1997).

To characterize task-specific activations, we used a model embodying 3 effects. The first simply examined task vs. baseline activations by contrasting all scans in which subjects were performing the grammaticality judgment with all those in which they were performing the sensorimotor, baseline task. The resulting SPM (t) was used to constrain the second and third analyses, which were concerned with describing learning-related effects. This constraint meant that we explored for learningrelated changes in activation only in those regions that showed an activation relative to the baseline. The second level of analysis examined task vs. baseline activations, which were expressed linearly within blocks with repeated item learning. The third analysis evaluated time-dependent (across block) modulation of these within-block linear changes as expressed over the entire duration of the experiment. We present results for linear changes alone. $\mathrm{H}$ owever, a separate analysis embodying more flexible 2 nd order effects was also carried out but did not add to the characterization of learning effects over and above those seen in a linear model. For all effects, subjects' data were modelled separately and the only activations presented are those expressed in all subjects, in all conditions, with no statistically significant differences in the magnitude of change among individual subjects. As our analysis was hypothesis driven, we present findings that survive an uncorrected (for the entire brain volume) threshold of $P<.01$.

\section{RESULTS}

\section{Behavioural Results}

As predicted, performance levels showed a linear improvement (see Fig. 3) both within blocks ( $D F=3 ; F=10.6 ; P<0.001$ ) and across blocks ( $D F=3 ; F=8 ; P=0.001$ ). There was no significant block by epoch interaction. A separate test on the first epoch of each block showed a significant improvement in classifying the novel items as the experiment evolved ( $D F=3$; $F=3.9 ; P<0.05)$. This observation provides evidence of progressive rule learning since in all cases, the first item of a block consisted entirely of novel items that could not be classified correctly on the basis of remembered instances.

\section{Functional Neuroimaging}

The effects of encoding novel grammatical items necessary for task performance were examined using two models. In the first model, we examined the effect of repeated presentation for the set of consonant strings presented in the first block alone where, due to the influence of trail by trial feedback, task performance is 


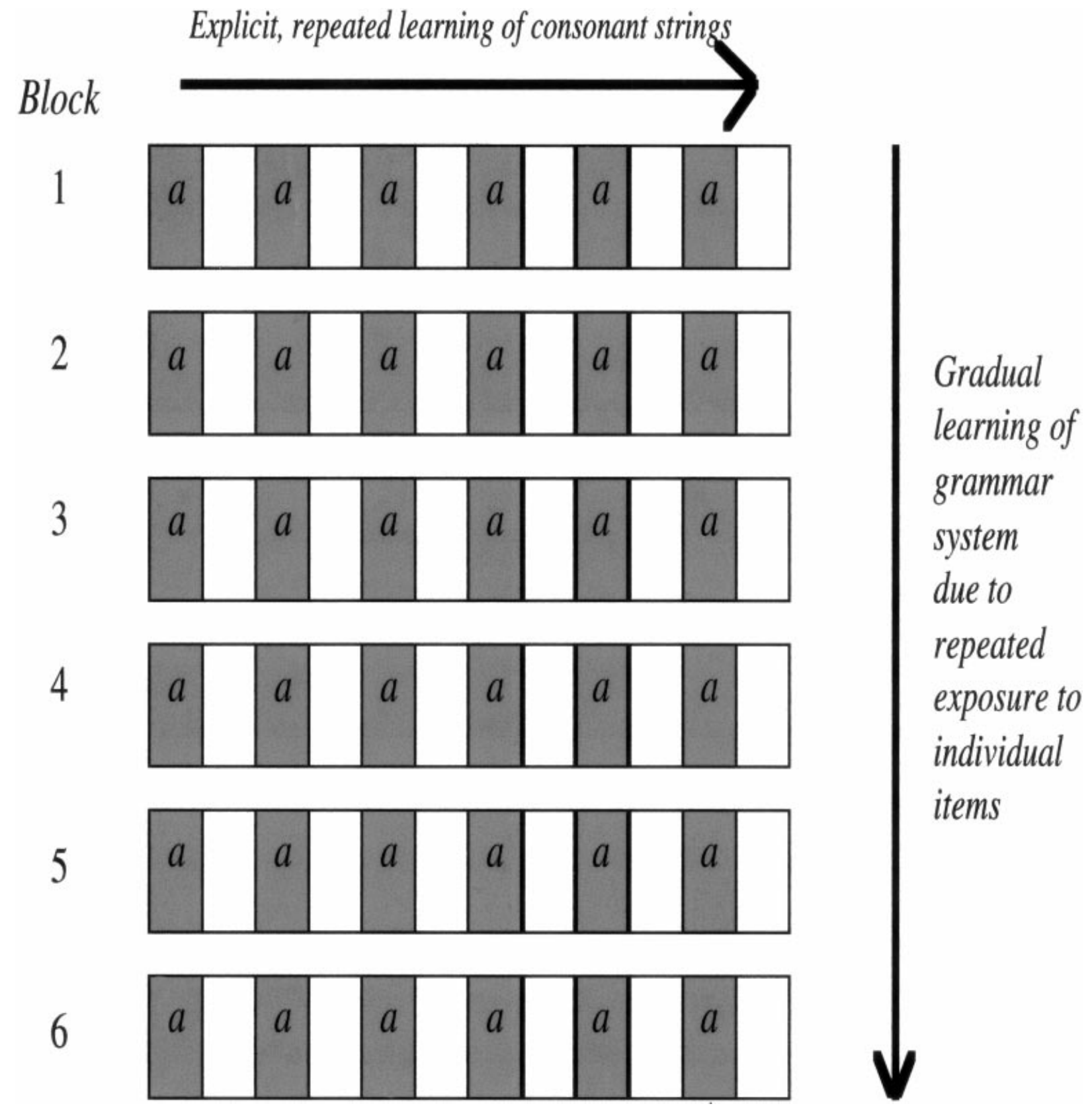

FIGURE 2. The overall design of the experiment. Red boxes represent epochs of activation condition and white boxes epochs of baseline. Each row represents a single block within which novel exemplars are presented and item-specific learning occurs within the block. Across the rows rule learning occurs as shown by behavioral data.

primarily dependent upon episodic memory. At this stage the influence of rule application, acquired across blocks, is minimal hence the effects emphasized early in the block reflect episodic encoding while effects later in the same block are biased towards episodic retrieval. These effects can also be conceived as reflecting decreasing novelty and increasing familiarity, respectively. With respect to our prediction of MTL activation at encoding, we found a significant left anterior hippocampal effect. Specifically, this region showed decreasing activation as a function of decreasing item novelty. This response pattern, within the first block, can be contrasted with an opposite response pattern seen in left posterior M TL where there was increased activation, as the block progressed. N ote that as the block progresses, subjects base their responses on retrieval of outcomes of previous responses to presentation of items. Consequently, we interpret this pattern of activation as reflecting episodic retrieval (see Fig. 4a).

The effects outlined above were ascertained from the first stimulus presentation block alone. 0 ur next analysis involved all 6 blocks and examined how encoding and retrieval responses are modulated by increasing knowledge of the grammatical rules. 


\section{Performance}

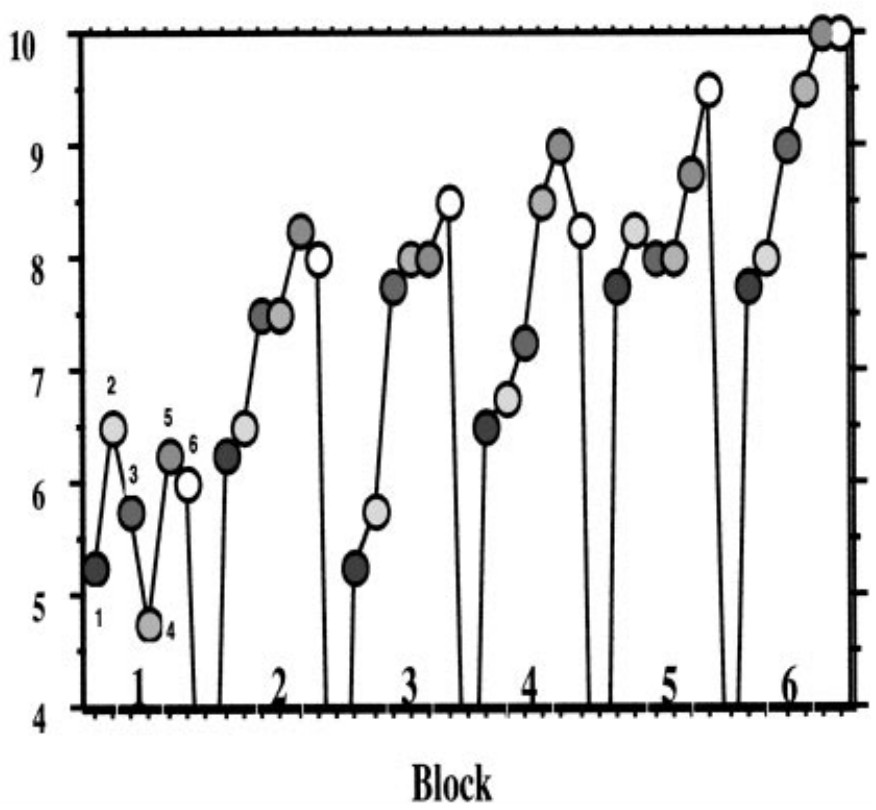

FIGURE 3. Mean of subjects performance within and across blocks. Within each block (from epoch 1 to epoch 6: these are labeled for block 1 only) there was an improvement in performance due to the repeated presentation of items and feedback as to their status. Consequently, this within-block effect reflect episodic memory primarily. The rule learning occurring from block to block is evidenced by the fact that there is a significant increase in performance on the first epoch of each successive block even though items seen in these cases were novel and the subject had received no prior knowledge of their grammatical status. This is evidence for a modulation of episodic memory by rule learning, which is expressed to greater degrees across blocks.

That is, we explored the data for regions in which an initial within-block increase in activation was attenuated or reversed with increasing familiarity with the grammar system and vice versa. We found that anterior and posterior regions of $M T L$ showed opposite effects with respect to this contrast. The anterior region (left hippocampus) showed a time-dependent reversal of the initial within-block relative increase, followed by adaptation, in activation as a response to initial presentation of new exemplars. The posterior MTL region (left parahippocampal gyrus) showed the opposite effect where a within-block progressively increased response to repeated presentation of exemplars was reversed in later blocks. In simple terms, what we demonstrate is encoding and retrieval-related neuronal responses that are modulated by psychological context or, more specifically, the acquisition of rule knowledge (see Fig. 4b).

\section{DISCUSSION}

This experiment examined two questions regarding the role of the hippocampus, and related medial temporal lobe structures, in episodic memory. Specifically, we asked whether dissociable functional roles could be established for distinct regions of M TL at episodic encoding and retrieval, respectively. We show an anterior hippocampal response that reflects the degree to which subjects actively encode exemplars of a modified artificial grammar system. The encoding-related response is localized to a region of left anterior hippocampus where we previously showed a hippocampal response in a paired-associate learning task ( $D$ olan and Fletcher, 1997). This anterior hippocampal response can be contrasted with a posterior M TL activation, located to parahippocampal gyrus, which is maximally responsive in situations where there is an emphasis on episodic retrieval.

Taken at face value, our data suggest a anterior-posterior functional segregation in the M TL with respect to encoding and retrieval. This anterior-posterior dissociation suggests that M TL is involved in both the encoding and retrieval stages of memory but that distinct regions of MTL are specialized for psychological operations engaged at these two stages. Lepage et al. (1998) in a recent meta-analysis of an extensive PET literature on medial temporal lobe activations in studies of episodic memory concluded that the empirical data suggest a rostral-caudal functional division within M TL. This functional division reflected a preponderance of studies reporting activation of anterior $M T L$ regions at encoding and posterior M TL regions at retrieval. O ur empirical data, derived from $\mathrm{fM} \mathrm{RI}$, accord entirely with the conclusions on this survey. H owever, Schacter and Wagner (1999, this issue), on the basis of their analysis of published $\mathrm{fMRI}$ data and a reconsideration of the evidence reviewed by Lepage et al. (1998) reach a different conclusion. These authors conclude that $\mathrm{fM} \mathrm{RI}$ evidence suggests that posterior M T L is associated with encoding and PET studies of encoding show both anterior and posterior M TL activations. $O n$ the basis of their conclusion, our findings of a rostral-caudal dissociation in hippocampal activations at encoding and retrieval would appear to be at odds with other fM RI experiments. Before discussing our own findings in greater detail, we first consider key fMRI studies reviewed by Schacter and Wagner (1999) and ask whether their conclusion of an encodingrelated memory specificity for posterior MTL activations is actually warranted.

Three early experiments, all of which used picture presentations, are similar in design and can be considered together. The earliest study is that of Stern et al. (1996) who reported posterior hippocampal activations in response to passive viewing of novel pictures presented over two separate experimental blocks. Likewise, Gabrieli et al. (1997) reported posterior M TL activation, with a focus in parahippocampal gyrus, in a similar type of experiment where subjects were presented with sets of pictures depicting everyday scenes with a task requirement to make an indoor-outdoor judgment. M ore recently, Rombouts et al. (1997) contrasted responses to pictures of scenes, which contained two objects whose names were written below the pictures, and a control condition in which the same scene was presented repeatedly. In this study, bilateral parahippocampal responses were reported in all but one subject with just over half the subjects also showing hippocampal activations. A common feature in all three studies is that the control condition involved repeated presenta- 
sagittal
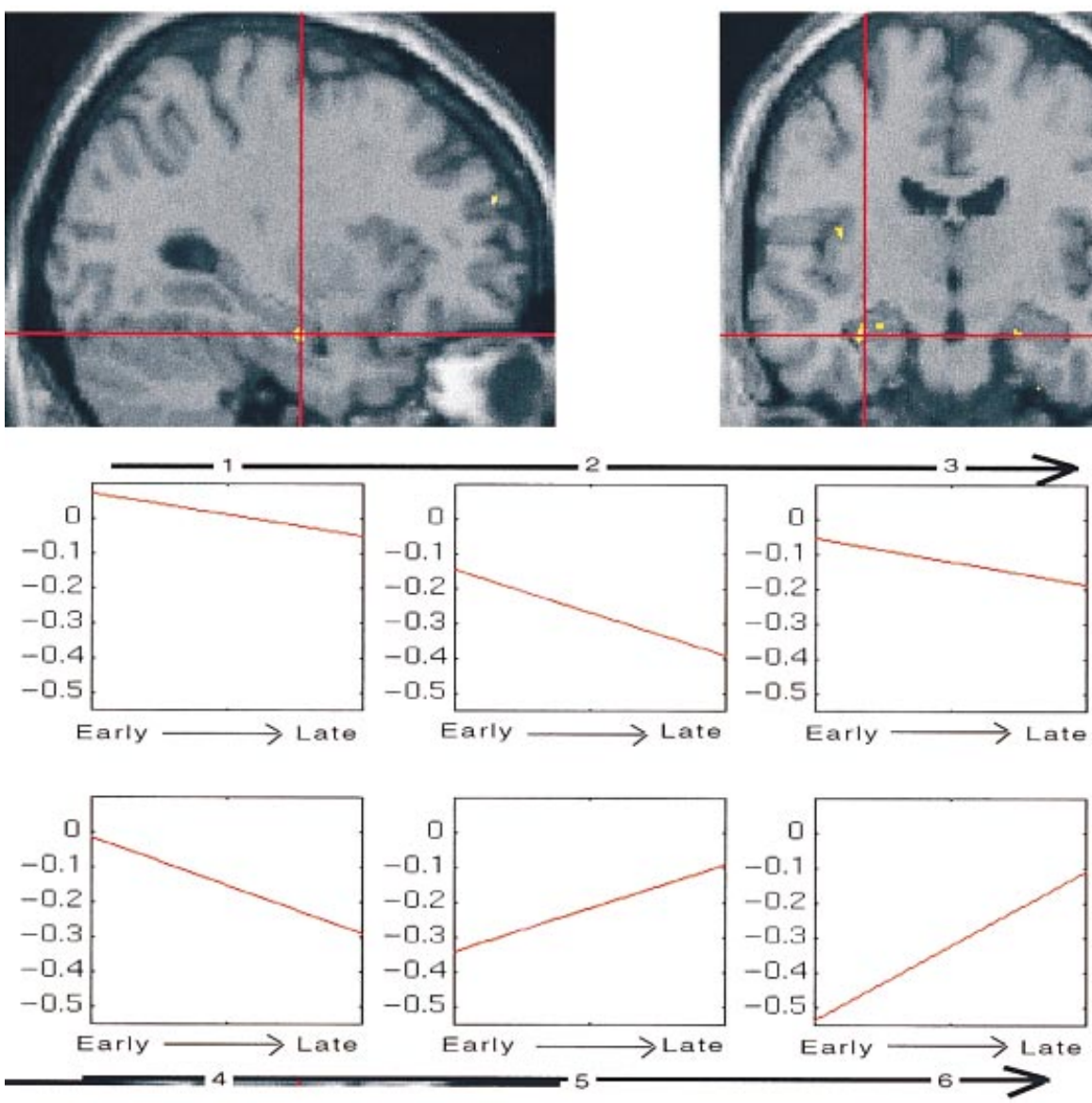

sagittal

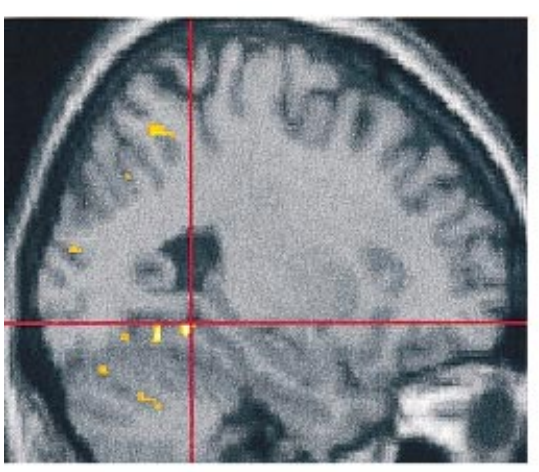

coronal
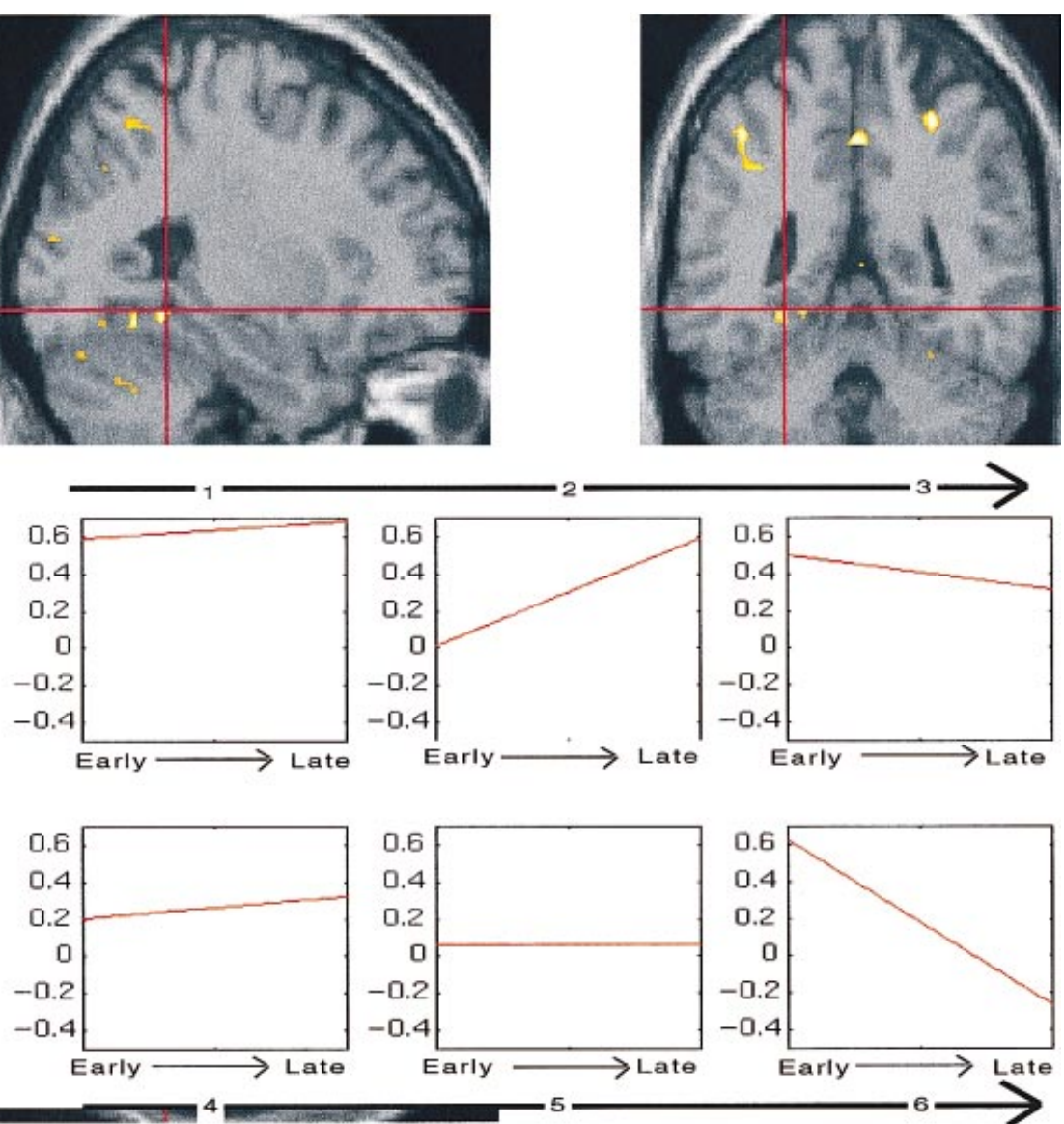

FIGURE 4 
tion of either one (Stern et al., 1996; Rombouts et al., 1998) or two (G abrieli et al., 1997) pictures. Consequently, in a subtractive approach the interpretation of differences between experimental and control conditions is problematic in that there is a possibility that what is actually being measured is adaptation effects to highly familiar scenes.

A more recent study by Fernandez at al. (1998) also reported posterior hippocampal activations in association with successful encoding of fifteen word lists. In a parametric experimental design, the authors correlated the number of successfully recalled words ascertained following a 15-second distraction period, with M TL activation at encoding. In this study, it is important to note that image volumes were acquired over a 15-second period corresponding to the presentation of 5 words and that correlations over the time-series were established for each separate 5 word list related volume. H owever, this design means that the first and third 5 word list volumes are biased towards primacy and recency effects with only the middle word-list volumes unambiguously reflecting long-term memory encoding. Unfortunately, no data are provided for an analysis based upon volumes representing this component of what is in effect the most sensitive, with respect to long-term memory, portion of a classic 15 word serial-position curve.

W hile in our view the studies outlined above do not provide a convincing basis for conclusions regarding the encoding-related MTL activations more recent experiments, using event-related fMRI paradigms, provide more challenging data. The use of event-related paradigms eschews a necessity for block stimulus presentation and allows sampling of hemodynamic responses to single stimulus presentation in mixed-trial designs. In two notable experiments, the common approach has been to determine whether the pattern of stimulus elicited activation at encoding predicts latter retrieval success. Brewer et al. (1998) presented subjects with pictures and reported that the magnitude of

FIGURE 4. TOp: SPM of regions showing a time by condition interaction with respect to an initial within-block reduction in activation. Top: Two orthogonal sections of a spatially normalized structural M RI scan with activations surviving a statistical threshold of $\mathbf{P}<.05$ (uncorrected) superimposed upon it. The sections were chosen at the left hippocampal maxima $(x, y, z=-30,-12,-12 ; z=$ 2.7). Bottom: Changes in activation (relative to the baseline task) at the early (first three presentation of exemplars) and late (last three presentations of exemplars) stages for each of the 6 blocks. The changing slope across blocks reflects the modulation of neural responses to novel exemplars by an emerging rule knowledge. Note the apparent deactivation of the hippocampus, with respect to baseline, for early presentation of items is a function of averaging response for the first three presentations of individual items (i.e., the ten exemplars presented threetimes). The thicker arrows (with blocks 1 to 6 labeled) represent increasing knowledge of and use of the rule system. The thin arrows represent progression from early to late learning within each block. b: SPM of regions showing a time by condition interaction with respect to an initial within-block increase in activation. Top: Two orthogonal sections of a spatially normalized structural M RI scan with activations surviving a statistical threshold of $P<.05$ (uncorrected) superimposed upon it. The sections were chosen at the left parahippocampal maxima $(x, y, z=-26,-46,-8$; $Z=2.8$ ). Bottom: Changes in activation are shown graphically as for Figure 3. activation in bilateral parahippocampal regions predicted subsequent retrieval success in a latter recognition task. H owever, the field of view in this study was restricted and did not encompass the anterior hippocampus while the use of everyday pictures raises a possibility that there may be cueing of associative episodic memory retrieval processes. Wagner et al. (1998) used a broadly similar strategy, in conjunction with word presentation and whole brain acquisition, where the experimental task was to make a semantic decision in relation to each individually presented word. In this study, subsequent recognition performance was predicted by the magnitude of the parahippocampal response during study. The specificity of the reported parahippocampal response rests on an assumption that encoding processes al one were engaged during scanning. The nature of the encoding task involving a necessity to perform a high level semantic judgment means that there may well be engagement of associative mnemonic processes that entail a degree of semantic memory retrieval. In other words, it can be argued that M TL activation might equally reflect engagement of retrieval processes. In our view, the interpretation of the findings in these studies raises fundamental issues as to optimal experimental designs for disambiguating encoding and retrieval related activations.

In our study, subjects were required to learn the grammatical status of novel exemplars from an artificial grammar system. A particular advantage of this paradigm is that it is possible to implement a within-subject design to examine encoding and retrieval processes in parallel without any change in the basic task instruction. In this regard, it needs to be emphasized that other studies that have addressed the issue of encoding-retrieval related functional specialization within MTL have not combined a within-subject design with the use of identical study material at encoding and retrieval (Gabrieli et al., 1997). Furthermore, as al ready outlined, the use of a modified artificial grammar learning paradigm provides a means of assessing how the emergence of an underlying knowledge system modulates episodic encoding and retrieval related activation effects. Recall that at the outset of a prototypical artificial grammar learning experiment subjects have no knowledge of the rule system and successful task performance requires exclusive reliance on episodic memory processes. O ver the course of an experiment, subjects acquire increasing knowledge of grammatical rules and consequently, with time, rule based (semantic) effects predominate in task performance. Consequently, over the course of an experiment, what is expressed is a dynamic interplay between episodic and rule (or semantic) related processing. W ithin this general framework our observation of left anterior hippocampal response, within the first block, to first presentation of exemplars emphasizes encoding processes that are not contaminated by rule based knowledge. The across-block timedependent attenuation of this encoding response mirrors a decreasing reliance on learning particular instances of the grammar (i.e., episodic encoding) and the emergence of rule-based task performance. In other words, the time by condition interaction for anterior hippocampal activation reflects a modulation of episodic encoding processes by the emergence of a rule-based system. This pattern contrasts with responses in posterior parahippocampus to increasing within-block presentation of exemplars. 
As for the response in anterior hippocampus, there was a time-dependent modulation of this within-block parahippocampal response. Note that in early phases of the experiment, increasing within-block presentation of items preferentially engages episodic retrieval processes that, with the acquisition of underlying rules, become less pronounced across blocks. Conse quently, the time-dependent modulation of this parahippocampal response, as for encoding related responses, mirrors subjects' increasing utilization of a ruled-based task performance strategy. In effect, what this aspect of our experiment demonstrates is a psychological modulation of episodic encoding and retrieval related neuronal responses, in anterior hippocampus and posterior parahippocampus, respectively, by an emerging ruled (or meaning) based system.

An important issue in studies of episodic memory concerns the psychological functions reflected in encoding and retrieval related neuronal responses. In previous work, we addressed the question of whether MTL responses at encoding reflects processing of stimulus novelty (D olan and Fletcher, 1997; Elliott and Dolan, 1998). M artin et al. (1997) reporting findings of anterior hippocampal activations that were sensitive to stimulus parameters (e.g., meaningfulness of items) and novelty, have drawn attention to different meanings attached to the concept of novelty. Thus, novelty can variously refer to an item's inherent meaning (nonsense items are more novel than real items), recency of occurrence of the item within an experimental context, and task experience (the first time a task is performed is more novel than subsequent task performance). The broader idea here is that novelty is a relative concept and reflects a dynamic between sensory features of the environment, past history of encounters with similar stimuli, and the degree to which such features can be accommodated within a meaning-based interpretative framework. This framework has particular relevance to our findings where the pattern of hippocampal response that we observed, within and across block, is amenable to a novelty-based interpretation. For example, the within-block attenuation of hippocampal response reflects a novelty effect in that it parallels increasing familiarity (or decreasing novelty) with individual exemplars of the grammar system. What needs explanation is the attenuation of this hippocampal novelty response across blocks as subjects acquired increasing knowledge of grammatical rules. 0 ur view is that the novelty of new exemplars is not equivalent across blocks in that, as the behavioral data demonstrate, increasing knowledge of the grammatical rules means that new exemplars of the grammar system can be processed with respect to the grammatical rules. From this perspective, the new exemplars are less novel and we propose that it is this influence of psychological context on exemplar processing that accounts for the across-block attenuation of a novelty response. $\mathrm{H}$ owever, it can equally be argued that these novelty effects can be explained on the basis of decreasing encoding demands with increasing within-block familiarity and an across-block reliance on rule-based task performance.

Although encoding and novelty detection are often conceptualized as independent processes (Fernandez et al., 1998), we are of the view that novelty detection is a fundamental aspect of encoding. This is also implicit in a model of memory function proposed by Shallice where novelty reflects situations where thought and action routines triggered by the environment are inadequate to produce an adaptive behavioral response (Shallice, 1988). N ovelty detection in this framework represents an early form of encoding whose role includes detection of adaptively significant events. It is self-evident that determining whether something is novel (as opposed to familiar) requires that incoming signals are compared with preexisting stored representations. Such an interaction in novelty assessment is compatible with recent extensions of the episodic memory concept, which is now conceptualized as arising out of an underlying semantic system (Tulving and Markowitsch, 1998). Novelty is likely to reflect situations with potential behavioral significance for an organism where formation of associated memory representations ensures that future encounters with similar stimuli will be both adaptive and less resource demanding.

0 ur theoretical position is that encoding involves a number of distinct psychological processes that are anatomically dissociable. At the most simple level, encoding can be described in terms of at least two different levels of stimulus analysis with the earliest level characterized by parallel analysis of elementary stimulus features and a second level involving more elaborative processing (Treisman and G elade, 1980). For example, an emphasis on processing semantic attributes of stimulus material has been consistently shown to activate a regions of left prefrontal cortex in the inferior frontal gyrus (Shallice et al., 1994; Kapur et al., 1994). We propose that establishing memory representations for totally unfamiliar items, particularly when they are not susceptible to semantic processing, emphasizes sensory-feature processing and that this is what is primarily reflected in anterior hippocampal activation. From this perspective, anterior hippocampal activation to novelty reflects a global integration of sensory stimuli with the current psychological context (H algren and M arinkovic, 1995).

The proposal that human functional neuroimaging data showing an anterior hippocampal response reflects novelty processing is also suggested by electrophysiological studies in humans and single cell recording studies in animals ( $\mathrm{H}$ algren and $\mathrm{M}$ arinkovic, 1995; M usen and Treisman, 1990; Gershberg and Shimamura, 1995). Direct single cell recordings from the hippocampus in patients has demonstrated units with preferential responses to novel as opposed to familiar stimuli (Fried et al., 1997). Recent neuropsychological data al so report the absence of a characteristic electrophysiological response to novelty in patients with hippocampal lesions (Knight, 1996). A sensitivity to novelty is al so implicit in the suggestion that hippocampal-dependent circuits maintain a template of the recent past for comparison with incoming stimuli (M artin et al., 1996).

Although functional neuroimaging provides a powerful tool for describing functional specialization within memory-related brain systems, it is nevertheless important to reconcile imaging data with what is observed in patients with circumscribed lesions. W ith respect to the $M T L$, the critical issue is whether the functional attributions of anterior and posterior activations explain findings in patients such as preserved semantic knowledge acquisition in 
young patients with hippocampal lesions (Vargha-Khadem et al., 1997) and the relatively restricted retrograde amnesia in adults with the same type of lesions (Reed and Squire, 1998). While accepting an absence of absolute anatomical specificity in lesion data and the preliminary nature of high quality functional neuroimaging data on $M T L$, we suggest that at the very least our data are consistent with current lesion data. An anterior hippocampus encoding/novelty specificity would be consistent with a relatively restricted retrograde amnesia seen in patients with lesions that primarily involve this region. In a similar vein, the ability of patients with hippocampal lesions to acquire semantic knowledge about the world is not inconsistent with our proposal that encoding is a multi-stage process in which the hippocampus mediates an early sensory-based stage that is necessary for subsequent recollective experience.

\section{ACKNOWLEDGMENTS}

R.J.D. and P.F. are supported by the WellcomeTrust.

\section{REFERENCES}

Brewer JB, Zhao Z, Desmond JE, Glover GH, Gabrieli JDE. 1998. $M$ aking memories: brain activity that predicts how well visual experience will be remembered. Science 281:1185-1187.

Cohen N J. 1984. Preserved learning capacity in amnesia: evidence for multiple memory systems. In: Squire LR, Butters N, editors. N europsychology of memory. N ew York: Guilford Press, p 83-103.

Dolan RJ, Fletcher PF. 1997. Dissociating prefrontal and hippocampal function in episodic memory. N ature 388:582-585.

Elliott R, Dolan RJ. 1998. Neural response during preference and memory judgments for subliminally presented stimuli: a functional neuroimaging study. J N eurosci 18:4697-4704.

Fernandez G, W eyerts H, Schrader-Bolsche M, Tendolkar I, Smid H GO , Templemann $C$, H inrichs $H$, Scheich $H$, Elger CE, M angun GR, $\mathrm{H}$ einze $\mathrm{H}$-J. 1998. Successful verbal encoding into episodic memory engages the posterior hippocampus: a parametrically analyzed functional magnetic resonance imaging study. J Neurosci 18:1841-1847.

Fletcher PC, Shallice T, Dolan RJ. 1998a. The functional roles of prefrontal cortexin episodic memory. I. Encoding. Brain 121:12391248.

Fletcher PC, Shallice T, Frith CD, Frackowiak RSJ, Dolan RJ. 1998b. The functional roles of prefrontal cortx in episodic memory. II. Retrieval. Brain 121:1249-1256.

Fried I, M acdonald KA, W ilson CL. 1997. Single neuron activity in hippocampus and amygdala during recognition of faces and objects. Neuron 18:875-887.

Friston KJ, Ashburner J, Frith CD , Poline JB, H eather JD, Frackowiak RSJ . 1995a. Spatial registration and normalization of images. H uman Brain M ap 2:165-189.

Friston KJ, Holmes AP, Worsley KJ, Poline J-P, Frith CD, Frackowiak RS) . 1995b. Statistical parametric mapping in functional imaging: a general linear approach. H uman Brain M ap 2:189-210.
Gabrieli JD, Brewer JB, D esmont JE, G lover G H . 1997. Separate neural bases of two fundamental memory processes in the human medial temporal lobe. Science 276:264-266.

Gershberg FB, Shimamura AP. 1995. Impaired use of organizational strategies in free recall following frontal lobe damage. N europsychologia 13:1305-1333.

$\mathrm{H}$ algren E, M arinkovic K. 1995. N europhysiological networks integrating human emotions. In: Gazzaniga MS, editor. The cognitive neurosciences. Cambridge, M A: M IT Press, p 1137-1380.

H olmes AP, Josephs O, Buchel C, Friston KJ. 1997. Statistical modelling of low frequency confounds in fM RI. N euroimage 5:S480.

Kapur S, Craik FIM , Tulving E, W ilson AA, H oule S, Brown G M . 1994. $N$ euroanatomical correlates of encoding in episodic memory: levels of processing effect. Proc N atl Acad Sci U SA 91:2008-2011.

Knight RT. 1996. Contribution of human hippocampal region to novelty detection. N ature 383:256-259.

Lepage M, H abib R, Tulving E. 1998. H ippocampal PET activations of memory encoding and retrieval: The HIPER model. H ippocampus 8:313-322.

M artin A, W iggs C, Ungerleider LC, H axby JC. 1996. Correlates of category-specific knowledge. N ature 379:649-652.

$M$ artin A, W iggs CL, Weisberg J. 1997. M odulation of human medial temporal lobe activity by form, meaning, and experience. H ippocampus 7:587-593.

Musen G, Treisman A. 1990. Implicit and explicit memory for visual patterns. J Exp Psychol 16:127-137.

Nyberg L, Cabeza R, Tulving E . 1996a. PET studies of encoding and retrieval: theH ERA model. Psychonom Bull Rev 3:135-148.

Nyberg L, M clntosh AR, Cabeza R, Habib R, H oule S, Tulving E. 1996b. General and specific brain regions involved in encoding and retrieval of events: what, where, and when. Proc N atl Acad Sci USA 93:11280-11285.

Reed JM, Squire LR. 1998. Retrograde amnesia for facts and events: findings from four new cases. J N eurosci 18:3943-3954.

Rempel-Clower N I, Zola SM , Squire LR, Amaral D G. 1996. Three cases of enduring memory impairment after bilateral damage limited to the hippocampal formation. J N eurosci 16:5233-5255.

Rombouts S, M achielsen W, Witter M, Barkhof F, Lindeboom J, Scheltens P. 1997. Visual association encoding activates the medial temporal lobe: a functional magnetic resonance imaging study. H ippocampus 7:594-601.

Rugg MD, Fletcher PC, Frith CD, Frackowiak RSJ, Dolan RJ. 1996. Differential activation of the prefrontal cortex in successful and unsuccessful memory retrieval. Brain 119:2073-2083.

Schacter DL, Wagner AD. 1999. Medial temporal lobe activation in fM RI and PET studies of episodic encoding and retrieval. H ippocampus 9:7-24.

Scoville W B, M ilner B. 1957. Loss of recent memory after bilateral hippocampal lesions. J N eurol N eurosurg Psychiatry 20:11-21.

ShalliceT. 1988. From neuropsychology to mental structure. C ambridge: Cambridge University Press.

Shallice T, Fletcher P, Frith CD, Grasby P, Frackowiak RSJ, D olan RJ. 1994. Brain regions associated with acquisition and retrieval of verbal episodic memory. N ature 368:633-635.

Stern CE, C orkin S, Gonzales G,R., Guimares AR, Baker JR, Jennings PJ, Carr CA, Sugiura RM, Vedantham V, Rosen B. 1996a. The hippocampal formation participates in novel picture encoding: evidence from functional magnetic resonance imaging. Proc $\mathrm{N}$ atl Acad Sci U SA 93:8660-8665.

Talairach J, Tournoux P. 1988. Co-planar stereotaxic atlas of the human brain. Stuttgart: GeorgeT hieme Verlag.

Treisman A, Gelade G. 1980. A feature integration theory of attention. Cognit Psychol 12:97-136.

Tulving E, M arkowitsch HJ. 1998. Episodic and declarative memory: role of the hippocampus. H ippocampus 8:198-204. 
Tulving E, Kapur S, Craik FIM, Moscovitch M, Houle S. 1994a. Hemispheric encoding/retrieval asymmetry in episodic memory: positron emission tomography findings. Proc Natl Acad Sci USA 91:2016-2020.

Tulving E, Kapur S, M arkovitsch HJ, Craik FIM , H abib R, Houle S. 1994b. N euroanatomical correlates of retrieval in episodic memory: auditory sentence recognition. Proc N atl Acad Sci U SA 91:20122015.

Tulving E, M arkowitsch H J, Kapur S, H abib R, H ouleS. 1994c. N ovelty encoding networks in the human brain: positron emission tomography data. N euroreport 5:2525-2528.
Tulving E, Markowitsch HJ, Craik FIM, Habib R, Houle S. 1996. Novelty and familiarity activations in PET studies of memory encoding and retrieval. Cereb Cortex 6:71-79.

Vargha-Khadem F, Gadian D G, Watkins KE, C onnelly A, Van Paesschen W, Mishkin M. 1997. Differential effects of early hippocampal pathology on episodic and semantic memory. Science 277:376-380.

Wagner AD, Schacter DL, Rotte M, Koustaal W, M aril A, Dale AM, Rosen BR, Buckner RL. 1998. Building memories: remembering and forgetting of verbal experiences as predicted by brain activity. Science 281:1188-1191. 